\section{Adjunctive transdermal oestradiol improves positive symptoms and general psychopathology in women with schizophrenia}

\section{QUESTION}

Question: Does adjunctive transdermal oestradiol improve symptoms in women with schizophrenia?

Patients: 102 women who met the diagnostic criteria (DSMIV) for schizophrenia, schizoaffective disorder (bipolar subtype excluded) or schizophreniform disorder. Main exclusions: currently in manic phase of illness; currently using hormonal therapy; menopausal symptoms; pregnant or breastfeeding; or serious medical illness.

Setting: Two general hospitals in Melbourne, Australia (inpatient and outpatient clinics); recruitment 1 January 2001 to 30 April 2004.

Intervention: Transdermal oestradiol $(100 \mu \mathrm{g} /$ day; $\mathrm{n}=56)$ or transdermal placebo $(n=46)$ for 28 days, in addition to usual antipsychotic therapy. The majority of women (77\%) were taking atypical antipsychotics, 17\% took typical antipsychotics and data were missing for $4 \%$. There were no differences between the groups in antipsychotic medication.

Outcomes: Positive, negative and general psychopathology symptoms (Positive and Negative Syndrome Scale (PANSS)) and adverse events (Adverse Symptoms Checklist). Symptoms were assessed at baseline and every week thereafter. Outcomes were analysed using repeated measures ANOVA.
Patient follow-up: $91 \%$ in the oestradiol group and $78 \%$ in the placebo group completed the study and had sufficient data for analysis.

\section{METHODS}

Design: Randomised controlled trial.

Allocation: Concealed.

Blinding: Double blind.

Follow-up period: 28 days (no follow-up post intervention).

\section{MAIN RESULTS}

Adjunctive oestradiol improved positive symptoms $(p=0.005)$ and general pathological symptoms $(p=0.01)$ compared with placebo but had no effect on negative symptoms $(p=0.2)$. There was no significant difference in adverse events between groups ( $p=0.49$ ), with adverse events reducing in both groups from baseline $(p<0.001)$. Two participants $(3.6 \%)$ withdrew from the oestradiol group because of menstrual bleeding.

\section{CONCLUSIONS}

Adjunctive oestradiol is effective in improving positive and general psychopathological symptoms in women with schizophrenia.

\section{ABSTRACTED FROM}

Kulkarni J, de Castella A, Fitzgerald $\mathrm{PB}$, et al. Estrogen in severe mental illness-A potential new treatment approach. Arch Gen Psychiatry 2008;65:955-60.

Correspondence to: Jayashri Kulkarni, Alfred Psychiatry Research Centre, The Alfred and Monash University, School of Psychology, Psychiatry and Psychological Medicine, The Alfred Hospital, First Floor, Old Baker Bldg, Commercial Road, Melbourne, Victoria, 3004, Australia; j.kukarni@alfred.org.au

Source of funding: Stanley Medical Research Institute and the National Health and Medical Research Council of Australia.
E pidemiological investigations have demonstrated that schizophrenia is expressed differently with regard to age at onset, severity of symptoms, course and outcome between genders. Oestrogens and other gonadal hormones influence human emotions, cognition and behaviour and might have an impact on schizophrenic symptomatology. Clinical and animal studies have demonstrated the protective effect of oestrogen in schizophrenia ${ }^{1}$ while previous open label and double blind, placebo controlled clinical trials had pointed out the beneficial effect on controlling psychotic symptoms in women receiving oestradiol as an adjunctive therapy to antipsychotics. $^{2}$

The present study indicates that addition of $100 \mathrm{~g}$ of transdermal oestradiol in a clinically heterogeneous (acutely ill, chronic, treatment resistant) sample of women with schizophrenia significantly improves the efficacy of antipsychotics to reduce positive symptoms in the short term (4 weeks). Women were of childbearing age with normal oestradiol levels; no significant adverse side effects were reported in the active treatment group. Notably, thorough analysis of the results (total PANSS total and positive symptoms scores) seems to show that the significant differences between groups might be mediated by lack of response to treatment in the control group. Unfortunately, the effect size of the reported clinical changes is not reported. The fact that no significant differences were found in negative symptom improvement is also somewhat disappointing. believe that the improvement in negative and cognitive symptoms is the real challenge that psychopharmacology should face in schizophrenia during the next few years.

The clinical heterogeneity of the sample and the short evaluative time span weaken the study's design and, therefore, generalisation of these findings to clinical practice. While it is not currently possible to recommend oestrogens as adjunctive therapy in schizophrenia, the available evidence is strong enough to justify the continuation of well designed studies to reveal what benefit oestrogens may bring to the treatment of schizophrenia.

Benedicto Crespo-Facorro, MD, PhD, Rocio PerezIglesias, MD, PhD, Ignacio Mata, MD, PhD

Centro de Investigacion Biomedica en Red en Salud Mental (CIBERSAM), Department of Psychiatry, University of Cantabria, Schizophrenia Research Program, Hospial Marques de Valdecilla, Santander, Spain

Competing interests: BCF has received unrestricted research funding from AstraZeneca, Pfizer, Bristol-Myers Squibb and Johnson \& Johnson, and consultant fees from Pfizer.

1. Chua WL, de Izquierdo SA, Kulkarni J, et al. Estrogen for schizophernia. Cochrane Database Syst Rev 2005;4: CD004719

2. Riecher-Rössler A, de Geyter $\mathrm{C}$. The forthcoming role of treatment with oestrogens in mental health. Swiss Med Wkly 2007;137:65-72. 